NUMERICAL ANALYSIS AND MATHEMATICAL MODELLING

BANACH CENTER PUBLICATIONS, VOLUME 29

INSTITUTE OF MATHEMATICS

POLISH ACADEMY OF SCIENCES

WARSZAWA 1994

\title{
ORTHOGONAL POLYNOMIALS \\ AND THE LANCZOS METHOD
}

\author{
C. BREZINSKI, H. SADOK \\ Laboratoire d'Analyse Numérique et d'Optimisation, UFR IEEA-M3 \\ Université des Sciences et Technologies de Lille \\ F-59655 Villeneuve d'Ascq Cedex, France \\ E-mail:BREZINSK@FRCITL81.BITNET \\ M. REDIVO ZAGLIA \\ Dipartimento di Elettronica e Informatica, Università degli Studi di Padova \\ Via Gradenigo 6/a, I-35131 Padova, Italy \\ E-mail:ELEN@ELETT1.DEI.UNIPD.IT
}

\begin{abstract}
Lanczos method for solving a system of linear equations is well known. It is derived from a generalization of the method of moments and one of its main interests is that it provides the exact answer in at most $n$ steps where $n$ is the dimension of the system. Lanczos method can be implemented via several recursive algorithms known as Orthodir, Orthomin, Orthores, Biconjugate gradient,... In this paper, we show that all these procedures can be explained within the framework of formal orthogonal polynomials. This theory also provides a natural basis for curing breakdown and near-breakdown in these algorithms. The case of the conjugate gradient squared method can be treated similarly.
\end{abstract}

1. Lanczos method. Let us consider in $\mathbb{C}^{n}$ the system of linear equations

$$
A x=b \text {. }
$$

The Lanczos method [14] for solving this system consists in constructing the sequence of vectors $\left(x_{k}\right)$ as follows:

- choose two arbitrary nonzero vectors $x_{0}$ and $y$,

1991 Mathematics Subject Classification: 65F10, 65F25.

Key words and phrases: Lanczos method, biconjugate gradient, projection, orthogonal polynomials.

The paper is in final form and no version of it will be published elsewhere. 
- set $r_{0}=b-A x_{0}$,

- determine $x_{k}$ such that

$$
\begin{gathered}
x_{k}-x_{0} \in E_{k}=\operatorname{span}\left(r_{0}, A r_{0}, \ldots, A^{k-1} r_{0}\right), \\
r_{k}=b-A x_{k} \perp F_{k}=\operatorname{span}\left(y, A^{*} y, \ldots, A^{*^{k-1}} y\right)
\end{gathered}
$$

where $A^{*}$ is the conjugate transpose of $A$.

These two conditions determine $x_{k}$ if it exists. Indeed, $x_{k}-x_{0}$ can be written in the form

$$
x_{k}-x_{0}=-\alpha_{1} r_{0}-\ldots-\alpha_{k} A^{k-1} r_{0}
$$

and the orthogonality conditions give

$$
\left(A^{*^{i}} y, r_{k}\right)=0 \quad \text { for } i=0, \ldots, k-1,
$$

which is a system of $k$ linear equations in the $k$ unknowns $\alpha_{1}, \ldots, \alpha_{k}$. This system is singular if $r_{0}, A r_{0}, \ldots, A^{k-1} r_{0}$ or $y, A^{*} y, \ldots, A^{*^{k-1}} y$ are linearly dependent.

If we set

$$
P_{k}(\xi)=1+\alpha_{1} \xi+\ldots+\alpha_{k} \xi^{k}
$$

then we have

$$
r_{k}=P_{k}(A) r_{0}
$$

Moreover, if we set

$$
c_{i}=\left(y, A^{i} r_{0}\right), \quad i=0,1, \ldots
$$

and if we define the linear functional $c$ on the space of polynomials by

$$
c\left(\xi^{i}\right)=c_{i}, \quad i=0,1, \ldots
$$

then the preceding orthogonality conditions can be written as

$$
c\left(\xi^{i} P_{k}\right)=0 \quad \text { for } i=0, \ldots, k-1 .
$$

These relations show that $P_{k}$ is a polynomial of degree at most $k$ belonging to the family of formal orthogonal polynomials with respect to $c$ [1]. This polynomial is defined apart from a multiplying factor which is chosen, in our case, such that $P_{k}(0)=1$. Due to this normalization, $P_{k}$ exists and is unique if and only if the Hankel determinant

is different from zero.

$$
H_{k}^{(1)}=\left|\begin{array}{cccc}
c_{1} & c_{2} & \ldots & c_{k} \\
c_{2} & c_{3} & \ldots & c_{k+1} \\
\vdots & \vdots & \ldots & \vdots \\
c_{k} & c_{k+1} & \ldots & c_{2 k-1}
\end{array}\right|
$$

The polynomials $P_{k}$ can be recursively computed in different ways which lead to the various Lanczos type algorithms known as Orthores, Orthodir, Orthomin, Biores, Biodir, biconjugate gradient, and so on. A unified presentation and derivation of all these methods can be based on the theory of formal orthogonal polynomials [8]. 
The main property of the Lanczos method is its finite convergence, namely that $\exists k \leq n$ such that $r_{k}=0$ and $x_{k}=x=A^{-1} b$.

The theory of the Lanczos method has its basis in generalizations of the method of moments and of Galerkin's method, as will now be explained.

2. Theory of the Lanczos method. Let $E$ be a vector space and $E^{*}$ its algebraic dual. Let $E_{k}=\operatorname{span}\left(u_{0}, \ldots, u_{k-1}\right)$ where $u_{0}, \ldots, u_{k-1} \in E$ are assumed to be linearly independent. Let $L_{0}, L_{1}, \ldots, L_{k-1}$ be $k$ linearly independent linear functionals in $E^{*}$.

Let $f \in E$ and let $R_{k} \in E_{k}$ be defined by

$$
\left\langle L_{i}, R_{k}\right\rangle=\left\langle L_{i}, f\right\rangle \quad \text { for } i=0, \ldots, k-1 .
$$

Let $H_{k}$ be the linear mapping on $E$ defined by

$$
H_{k} f=R_{k} \text {. }
$$

Then $H_{k} R_{k}=R_{k}$ and thus $H_{k}^{2}=H_{k}$, which shows that $H_{k}$ is a projection on $E_{k}$.

The method of moments, introduced by Vorobyev [16], is based on orthogonal projections in a Hilbert space. We shall now give its generalization as presented in $[3]$.

It consists in constructing a linear mapping $A_{k}$ on $E_{k}$ such that

$$
\begin{aligned}
u_{1} & =A_{k} u_{0}, \\
u_{2} & =A_{k} u_{1}=A_{k}^{2} u_{0}, \\
\ldots \ldots \ldots \ldots \ldots \ldots \ldots \ldots \ldots \ldots \ldots & \ldots \ldots \\
u_{k-1} & =A_{k} u_{k-2}=A_{k}^{k-1} u_{0}, \\
H_{k} u_{k} & =A_{k} u_{k-1}=A_{k}^{k} u_{0}
\end{aligned}
$$

with $H_{k}$ as defined above. These relations completely determine the mapping $A_{k}$. Since $H_{k} u_{k} \in E_{k}$, we can write

$$
H_{k} u_{k}=-\beta_{0} u_{0}-\ldots-\beta_{k-1} u_{k-1},
$$

that is,

$$
\beta_{0} u_{0}+\ldots+\beta_{k-1} u_{k-1}+H_{k} u_{k}=\left(\beta_{0}+\beta_{1} A_{k}+\ldots+\beta_{k-1} A_{k}^{k-1}+A_{k}^{k}\right) u_{0}=0 .
$$

But we have

$$
\left\langle L_{i}, u_{k}-H_{k} u_{k}\right\rangle=0 \quad \text { for } i=0, \ldots, k-1,
$$

that is,

$$
\beta_{0}\left\langle L_{i}, u_{0}\right\rangle+\ldots+\beta_{k-1}\left\langle L_{i}, u_{k-1}\right\rangle+\left\langle L_{i}, u_{k}\right\rangle=0 \quad \text { for } i=0, \ldots, k-1 .
$$

Thus, if we set

$$
\widetilde{P}_{k}(\xi)=\beta_{0}+\ldots+\beta_{k-1} \xi^{k-1}+\xi^{k}
$$

then it can be proved that

$$
\widetilde{P}_{k}\left(A_{k}\right) u_{0}=0 .
$$


Let us assume that $A_{k}$ is invertible in $E_{k}$ and solve the equation

$$
A_{k} v_{k}=u_{0}
$$

where $v_{k} \in E_{k}$.

Let $P_{k}$ and $V_{k-1}$ be two arbitrary polynomials of degree $k$ and $k-1$ respectively, related by

$$
1-P_{k}(\xi)=\xi V_{k-1}(\xi) .
$$

Thus $P_{k}$ must satisfy $P_{k}(0)=1$ and we have

$$
u_{0}-P_{k}\left(A_{k}\right) u_{0}=A_{k} V_{k-1}\left(A_{k}\right) u_{0} .
$$

If we take

$$
P_{k}(\xi)=\widetilde{P}_{k}(\xi) / \widetilde{P}_{k}(0)
$$

then

It follows that

$$
P_{k}(0)=1 \quad \text { and } \quad P_{k}\left(A_{k}\right) u_{0}=0 .
$$

which shows that

$$
A_{k} V_{k-1}\left(A_{k}\right) u_{0}=u_{0}
$$

$$
v_{k}=V_{k-1}\left(A_{k}\right) u_{0} .
$$

Let us now make the particular choice $E_{k}=\mathbb{C}^{n}$ and

$$
u_{i}=A^{i} r_{0}, \quad i=0, \ldots, k,
$$

where $A$ is an $n \times n$ regular matrix.

Let $A_{k}$ be the matrix obtained by the method of moments and let $x_{k}$ be the vector defined by

$$
A_{k}\left(x_{k}-x_{0}\right)=r_{0} .
$$

The linear functional $L_{i}$ is uniquely represented by a vector $y_{i}$ and

$$
\left\langle L_{i}, u_{j}\right\rangle=\left(y_{i}, u_{j}\right)
$$

where $(\cdot, \cdot)$ denotes the usual scalar product in $\mathbb{C}^{n} . H_{k}$ is the oblique projection on $E_{k}$ along $F_{k}^{\perp}$ where $F_{k}=\operatorname{span}\left(y_{0}, \ldots, y_{k-1}\right)$. and

The polynomial $P_{k}$ defined above is the same as that of the preceding section

$$
x_{k}-x_{0}=V_{k-1}\left(A_{k}\right) r_{0} .
$$

But, since $V_{k-1}$ has degree $k-1$ and $A^{i} r_{0}=A_{k}^{i} r_{0}$ for $i=0, \ldots, k-1$, we have

$$
x_{k}-x_{0}=V_{k-1}(A) r_{0} .
$$

That is,

$$
A x_{k}-b-\left(A x_{0}-b\right)=A V_{k-1}(A) r_{0}
$$

or

$$
r_{k}=r_{0}-A V_{k-1}(A) r_{0}=P_{k}(A) r_{0}
$$


Moreover, from the preceding results, we have

$$
\left(y_{i}, r_{k}\right)=0 \quad \text { for } i=0, \ldots, k-1,
$$

which is exactly the Lanczos method if $y_{i}=A^{*^{i}} y$ for $i=0, \ldots, k-1$. For more details, see [2].

Let us now look at the various possibilities for computing recursively the orthogonal polynomials $P_{k}$.

3. Orthogonal polynomials. We shall first consider the case where $\forall k, H_{k}^{(1)}$ $\neq 0$.

Let us define the linear functional $c^{(1)}$ by

$$
c^{(1)}\left(\xi^{i}\right)=c\left(\xi^{i+1}\right)=c_{i+1}, \quad i=0,1, \ldots
$$

Let $\left\{P_{k}^{(1)}\right\}$ be the family of monic orthogonal polynomials with respect to the linear functional $c^{(1)} . P_{k}^{(1)}$ exists under the condition that $H_{k}^{(1)} \neq 0$. As previously seen, this condition also ensures the existence of $P_{k} .\left\{P_{k}\right\}$ and $\left\{P_{k}^{(1)}\right\}$ are said to be adjacent families of formal orthogonal polynomials.

Since the polynomials of both families are uniquely determined, it is easy to see that

$$
P_{k+1}(\xi)=P_{k}(\xi)-\lambda_{k} \xi P_{k}^{(1)}(\xi)
$$

with $P_{0}(\xi)=P_{0}^{(1)}(\xi)=1$.

We now show how to obtain an expression for $\lambda_{k}$. Let $\left\{U_{i}\right\}$ be an arbitrary family of polynomials such that $\forall i, U_{i}$ has exact degree $i$. Multiplying both sides of (1) by $U_{k}$ and applying the functional $c$, we obtain

$$
\lambda_{k}=c\left(U_{k} P_{k}\right) / c\left(\xi U_{k} P_{k}^{(1)}\right) .
$$

Moreover, it is well known that a family of orthogonal polynomials satisfies a three-term recurrence relationship. Thus we have

$$
P_{k}^{(1)}(\xi)=\left(\xi-a_{k}\right) P_{k-1}^{(1)}(\xi)-b_{k} P_{k-2}^{(1)}(\xi)
$$

with $P_{0}^{(1)}(\xi)=1$ and $P_{-1}^{(1)}(\xi)=0$.

As proved in [4], using again the auxiliary family $\left\{U_{i}\right\}$ we have, by a similar argument,

$$
\begin{aligned}
b_{k} & =c\left(\xi^{2} U_{k-2} P_{k-1}^{(1)}\right) / c\left(\xi U_{k-2} P_{k-2}^{(1)}\right), \\
a_{k} & =\left[c\left(\xi^{2} U_{k-1} P_{k-1}^{(1)}\right)-b_{k} c\left(\xi U_{k-1} P_{k-2}^{(1)}\right)\right] / c\left(\xi U_{k-1} P_{k-1}^{(1)}\right) .
\end{aligned}
$$

For example, if we choose $U_{k}=P_{k}^{(1)}$, we recover the usual formula

$$
a_{k}=c\left(\xi^{2} P_{k-1}^{(1)^{2}}\right) / c\left(\xi P_{k-1}^{(1)^{2}}\right) .
$$


The family $\left\{P_{k}\right\}$ also satisfies a three-term recurrence relationship which can be written as

$$
P_{k+1}(\xi)=-D_{k}\left[\left(\xi-B_{k}\right) P_{k}(\xi)-C_{k} P_{k-1}(\xi)\right]
$$

with $P_{0}(\xi)=1$ and $P_{-1}(\xi)=0$. Since $P_{k}(0)=1$, we must have

$$
D_{k}=1 /\left(B_{k}+C_{k}\right) \text {. }
$$

Using again the auxiliary family $\left\{U_{i}\right\}$, as above, we have

$$
c\left(U_{k-1} P_{k+1}\right)=0=-D_{k}\left[c\left(\xi U_{k-1} P_{k}\right)-C_{k} c\left(U_{k-1} P_{k-1}\right)\right]
$$

and thus

$$
C_{k}=c\left(\xi U_{k-1} P_{k}\right) / c\left(U_{k-1} P_{k-1}\right) .
$$

Similarly,

$$
c\left(U_{k} P_{k+1}\right)=0=-D_{k}\left[c\left(\xi U_{k} P_{k}\right)-B_{k} c\left(U_{k} P_{k}\right)-C_{k} c\left(U_{k} P_{k-1}\right)\right]
$$

and thus we obtain

$$
B_{k}=\left[c\left(\xi U_{k} P_{k}\right)-C_{k} c\left(U_{k} P_{k-1}\right)\right] / c\left(U_{k} P_{k}\right) .
$$

Again, for the particular choice $U_{k}=P_{k}$, we recover the usual formulae.

Assume that $H_{k}^{(0)} \neq 0$ and set

$$
Q_{k}(\xi)=(-1)^{k} H_{k}^{(0)} P_{k}^{(1)}(\xi) / H_{k}^{(1)} .
$$

Since $P_{k}$ and $P_{k}^{(1)}$ both have degree $k$ exactly, the coefficients of $\xi^{k}$ in $P_{k}$ and $Q_{k}$ are the same and $Q_{k}$ is proportional to $P_{k}^{(1)}$. Moreover,

$$
c\left(\xi^{i+1} Q_{k}\right)=0 \quad \text { for } i=0, \ldots, k-1 .
$$

By using the same uniqueness argument as above we can write

$$
\begin{aligned}
Q_{k}(\xi) & =P_{k}(\xi)+\alpha_{k} Q_{k-1}(\xi), \\
P_{k+1}(\xi) & =P_{k}(\xi)-\beta_{k} \xi Q_{k}(\xi)
\end{aligned}
$$

with

$$
\alpha_{k}=-c\left(\xi U_{k-1} P_{k}\right) / c\left(\xi U_{k-1} Q_{k-1}\right) \quad \text { and } \quad \beta_{k}=c\left(U_{k} P_{k}\right) / c\left(\xi U_{k} Q_{k}\right) .
$$

4. Lanczos type algorithms. We shall now examine the various possibilities for computing recursively the vectors $r_{k}$ defined by $r_{k}=P_{k}(A) r_{0}$. They give rise to the different methods which are known and some new ones can also be obtained as we shall see below.

4.1. Lanczos/Orthodir. Let us set

$$
z_{k}=P_{k}^{(1)}(A) r_{0} .
$$

From (1) we immediately obtain

$$
r_{k+1}=r_{k}-\lambda_{k} A z_{k} .
$$


Using $r_{k}=b-A x_{k}$, this gives

$$
x_{k+1}=x_{k}+\lambda_{k} z_{k} .
$$

From (2) we have

$$
z_{k}=A z_{k-1}-a_{k} z_{k-1}-b_{k} z_{k-2} .
$$

This method is called Lanczos/Orthodir.

Let us now see how to compute the coefficients $\lambda_{k}, a_{k}$ and $b_{k}$ appearing in these formulae or, in other words, how to choose the auxiliary polynomials $\left\{U_{i}\right\}$.

The simplest choice consists in taking

$$
U_{k}(\xi)=\xi^{k}
$$

We have

$$
\lambda_{k}=c\left(\xi^{k} P_{k}\right) / c\left(\xi^{k+1} P_{k}^{(1)}\right)
$$

But

$$
c\left(\xi^{k} P_{k}\right)=\left({A^{*}}^{k} y, P_{k}(A) r_{0}\right) \quad \text { and } \quad c\left(\xi^{k+1} P_{k}^{(1)}\right)=\left(A^{*^{k+1}} y, P_{k}^{(1)}(A) r_{0}\right)
$$

and we finally obtain

$$
\lambda_{k}=\left(A^{*^{k}} y, r_{k}\right) /\left(A^{*^{k+1}} y, z_{k}\right) .
$$

Similarly, we have

$$
b_{k}=\left(A^{*^{k}} y, z_{k-1}\right) /\left(A^{*^{k-1}} y, z_{k-2}\right)
$$

and

$$
a_{k}=\left[\left(A^{*^{k}} y, A z_{k-1}\right)-b_{k}\left(A^{*^{k-1}} y, A z_{k-2}\right)\right] /\left(A^{*^{k}} y, z_{k-1}\right) .
$$

Now let us make the choice

$$
U_{k}(\xi)=P_{k}^{(1)}(\xi) .
$$

We have

Thus if we set

$$
\lambda_{k}=c\left(P_{k}^{(1)} P_{k}\right) / c\left(\xi P_{k}^{(1)^{2}}\right)
$$

$$
\widetilde{z}_{k}=\bar{P}_{k}^{(1)}\left(A^{*}\right) y=P_{k}^{(1)}(A)^{*} y
$$

we obtain

$$
\lambda_{k}=\left(\widetilde{z}_{k}, r_{k}\right) /\left(\widetilde{z}_{k}, A z_{k}\right) .
$$

We also have

$$
a_{k}=\frac{c\left(\xi^{2} P_{k-1}^{(1)^{2}}\right)}{c\left(\xi P_{k-1}^{(1)^{2}}\right)}=\frac{\left(A^{*} \widetilde{z}_{k-1}, A z_{k-1}\right)}{\left(\widetilde{z}_{k-1}, A z_{k-1}\right)}
$$

and then

$$
b_{k}=\frac{c\left(\xi^{2} P_{k-2}^{(1)} P_{k-1}^{(1)}\right)}{c\left(\xi P_{k-2}^{(1)^{2}}\right)}=\frac{\left(A^{*} \widetilde{z}_{k-2}, A z_{k-1}\right)}{\left(\widetilde{z}_{k-2}, A z_{k-2}\right)}
$$


But $c\left(\xi^{2} P_{k-2}^{(1)} P_{k-1}^{(1)}\right)=c\left(\xi P_{k-1}^{(1)^{2}}\right)$ by the orthogonality of $P_{k-1}^{(1)}$ to any polynomial of degree strictly less than $k-1$, and thus

$$
b_{k}=\left(\widetilde{z}_{k-1}, A z_{k-1}\right) /\left(\widetilde{z}_{k-2}, A z_{k-2}\right) \text {. }
$$

Thus, we obtain

$$
\widetilde{z}_{k}=A^{*} \widetilde{z}_{k-1}-\bar{a}_{k} \widetilde{z}_{k-1}-\bar{b}_{k} \widetilde{z}_{k-2}
$$

If we set

$$
\widetilde{r}_{k}=P_{k}(A)^{*} r_{0}
$$

then we have

$$
\widetilde{r}_{k+1}=\widetilde{r}_{k}-\bar{\lambda}_{k} A^{*} \widetilde{z}_{k}
$$

If we gather all these formulae together, we obtain the algorithm known under the name of BIODIR [12].

R e m a r k. This algorithm is defined (that is, $x_{k+1}$ exists) only if $P_{k}^{(1)}$ has exact degree $k$, or, in other words, if $\forall k, H_{k}^{(1)} \neq 0$. If this condition is not satisfied, it is possible to jump over the non-existing polynomials $P_{k}^{(1)}$ and to use only those which exist. Thus we obtain a generalization of BIODIR without breakdown. This algorithm, called the MRZ (Method of Recursive Zoom), was given in [6]. Related algorithms can also be found in [12]. They will be briefly described in Section 5 .

4.2. Lanczos/Orthores. Let us now assume that $P_{k}$ has exact degree $k$. Thus the three-term recurrence relationship (3) holds and we immediately obtain

$$
r_{k+1}=-D_{k}\left(A r_{k}-B_{k} r_{k}-C_{k} r_{k-1}\right) \text {. }
$$

Since $r_{k}=b-A x_{k}$, we have

$$
x_{k+1}=D_{k}\left(r_{k}+B_{k} x_{k}+C_{k} x_{k-1}\right) .
$$

These formulae define the method known under the name of Lanczos/Orthores (see $[17]$ ).

Let us now see how to compute the coefficients $D_{k}, B_{k}$ and $C_{k}$ appearing in these formulae. There are several possibilities according to the choice of the auxiliary polynomials $\left\{U_{i}\right\}$.

Let us start again by taking

$$
U_{k}(\xi)=\xi^{k}
$$

We have

$$
C_{k}=c\left(\xi^{k} P_{k}\right) / c\left(\xi^{k-1} P_{k-1}\right)
$$

and hence

$$
C_{k}=\left(A^{*^{k}} y, r_{k}\right) /\left(A^{*^{k-1}} y, r_{k-1}\right) .
$$

Similarly, we have

$$
B_{k}=\left[c\left(\xi^{k+1} P_{k}\right)-C_{k} c\left(\xi^{k} P_{k-1}\right)\right] / c\left(\xi^{k} P_{k}\right),
$$


that is,

$$
B_{k}=\left[\left(A^{*^{k}} y, A r_{k}\right)-C_{k}\left(A^{*^{k-1}} y, A r_{k-1}\right)\right] /\left(A^{*^{k}} y, r_{k}\right) .
$$

Since $P_{k}$ has exact degree $k$, we can also make the choice

$$
U_{k}(\xi)=P_{k}(\xi) \text {. }
$$

In that case

$$
C_{k}=c\left(\xi P_{k-1} P_{k}\right) / c\left(P_{k-1}^{2}\right) .
$$

But, multiplying both sides of (3) by $P_{k+1}$ and applying $c$, we obtain

$$
c\left(P_{k+1}^{2}\right)=-D_{k} c\left(\xi P_{k} P_{k+1}\right)
$$

and thus

$$
C_{k}=-\frac{1}{D_{k-1}} \frac{c\left(P_{k}^{2}\right)}{c\left(P_{k-1}^{2}\right)} .
$$

If we set

$$
\widetilde{r}_{k}=\bar{P}_{k}\left(A^{*}\right) y
$$

then

We also have, since $c\left(P_{k-1} P_{k}\right)=0$,

$$
C_{k}=-\frac{1}{D_{k-1}} \frac{\left(\widetilde{r}_{k}, r_{k}\right)}{\left(\widetilde{r}_{k-1}, r_{k-1}\right)} .
$$

$$
B_{k}=c\left(\xi P_{k}^{2}\right) / c\left(P_{k}^{2}\right),
$$

that is,

Finally, (3) gives us

$$
B_{k}=\left(\widetilde{r}_{k}, A r_{k}\right) /\left(\widetilde{r}_{k}, r_{k}\right)
$$

$$
\widetilde{r}_{k+1}=-\bar{D}_{k}\left(A^{*} \widetilde{r}_{k}-\bar{B}_{k} \widetilde{r}_{k}-\bar{C}_{k} \widetilde{r}_{k-1}\right) .
$$

These formulae define the algorithm known under the name of BIORES.

Rem ark. The two algorithms described in this subsection need the supplementary assumption that $P_{k}$ has exact degree $k$. Thus, for all $k, H_{k}^{(0)}$ and $H_{k}^{(1)}$ both have to be different from zero. The supplementary assumption $H_{k}^{(0)} \neq 0$ is needed by the procedure used in the recursive computation but it is, in fact, an unnecessary assumption in the theory of the Lanczos method and it is a supplementary reason for a breakdown. A remedy will be indicated in Section 5 .

4.3. Lanczos/Orthomin. Instead of using the polynomials $P_{k}^{(1)}$ as in the method Lanczos/Orthores, we shall make use of the polynomials $Q_{k}$ defined in Section 3. Thus $P_{k}$ is again assumed to have exact degree $k$. We set

$$
p_{k}=Q_{k}(A) r_{0} \text {. }
$$

From (4) and (5) we have

$$
p_{k}=r_{k}+\alpha_{k} p_{k-1} \text { and } r_{k+1}=r_{k}-\beta_{k} A p_{k} .
$$


By using the definition of $r_{k}$, it follows that

$$
x_{k+1}=x_{k}+\beta_{k} p_{k} .
$$

These formulae define the method known under the name of Lanczos/ Orthomin (see [11]).

Let us now see how to compute the coefficients $\alpha_{k}$ and $\beta_{k}$ appearing in these formulae. We begin by the choice

$$
U_{k}(\xi)=\xi^{k}
$$

We have

$$
\alpha_{k}=-c\left(\xi^{k} P_{k}\right) / c\left(\xi^{k} Q_{k-1}\right), \quad \beta_{k}=c\left(\xi^{k} P_{k}\right) / c\left(\xi^{k+1} Q_{k}\right)
$$

and it follows that

$$
\alpha_{k}=-\left(A^{*^{k}} y, r_{k}\right) /\left(A^{*^{k}} y, p_{k-1}\right), \quad \beta_{k}=\left(A^{*^{k}} y, r_{k}\right) /\left(A^{*^{k+1}} y, p_{k}\right) .
$$

Let us now choose

$$
U_{k}(\xi)=P_{k}(\xi)
$$

In that case

$$
\beta_{k}=c\left(P_{k}^{2}\right) / c\left(\xi P_{k} Q_{k}\right) .
$$

But, since the coefficients of $\xi^{k}$ in $P_{k}$ and in $Q_{k}$ are the same, we have

$$
P_{k}(\xi)=Q_{k}(\xi)+p(\xi)
$$

where $p$ is a polynomial of degree $k-1$ at most. Thus

$$
c\left(\xi P_{k} Q_{k}\right)=c\left(\xi Q_{k}^{2}\right)+c\left(\xi p Q_{k}\right) .
$$

But, as $Q_{k}$ is proportional to $P_{k}^{(1)}$, we have, by the orthogonality property of $P_{k}^{(1)}$, $c\left(\xi p Q_{k}\right)=0$ and thus

Setting

$$
\beta_{k}=c\left(P_{k}^{2}\right) / c\left(\xi Q_{k}^{2}\right)
$$

we obtain

$$
\widetilde{r}_{k}=P_{k}(A)^{*} y
$$

We have

$$
\beta_{k}=\left(\widetilde{r}_{k}, r_{k}\right) /\left(\widetilde{p}_{k}, A p_{k}\right)
$$

But $\beta_{k-1}=c\left(P_{k-1}^{2}\right) / c\left(\xi P_{k-1} Q_{k-1}\right)$ and thus

$$
\alpha_{k}=-c\left(\xi P_{k-1} P_{k}\right) / c\left(\xi P_{k-1} Q_{k-1}\right) .
$$

$$
\alpha_{k}=-\beta_{k-1} c\left(\xi P_{k-1} P_{k}\right) / c\left(P_{k-1}^{2}\right) \text {. }
$$

On the other hand, writing (5) for the index $k$, multiplying it by $P_{k}$ and applying $c$ gives

Moreover, from (4)

$$
c\left(P_{k}^{2}\right)=-\beta_{k-1} c\left(\xi P_{k} Q_{k-1}\right) .
$$

$$
c\left(\xi P_{k} Q_{k-1}\right)=c\left(\xi P_{k} P_{k-1}\right)+\alpha_{k} c\left(\xi P_{k} Q_{k-2}\right) .
$$


Since $P_{k}$ is orthogonal to $\xi Q_{k-2}$ which is a polynomial of degree $k-1$, it follows that

$$
c\left(P_{k}^{2}\right)=-\beta_{k-1} c\left(\xi P_{k} P_{k-1}\right)
$$

Thus, we finally obtain

$$
\alpha_{k}=c\left(P_{k}^{2}\right) / c\left(P_{k-1}^{2}\right)
$$

that is,

$$
\alpha_{k}=\left(\widetilde{r}_{k}, r_{k}\right) /\left(\widetilde{r}_{k-1}, r_{k-1}\right) \text {. }
$$

If we set

$$
\widetilde{p}_{k}=Q_{k}(A)^{*} y
$$

we obtain from (5)

$$
\widetilde{r}_{k+1}=\widetilde{r}_{k}-\bar{\beta}_{k} A^{*} \widetilde{p}_{k}
$$

and from (4)

$$
\widetilde{p}_{k}=\widetilde{r}_{k}+\bar{\alpha}_{k} \widetilde{p}_{k-1} .
$$

These formulae define the so-called biconjugate gradient method (BCG) due to Lanczos [14], but popularized by Fletcher [11]. When the matrix $A$ is Hermitian and when $y=r_{0}$ this method reduces to the conjugate gradient method of Hestenes and Stiefel [13].

4.4. Other methods. All the methods are characterized by the choice of one or two recurrence relationships for computing the orthogonal polynomials. There are many other possible choices, thus leading to other (new) methods. Some of them are described in [8].

5. Breakdown and near-breakdown. Let us now assume that some of the Hankel determinants $H_{k}^{(1)}$ are equal to zero. In that case some of the polynomials $P_{k}$, and thus the corresponding polynomials $P_{k}^{(1)}$, do not exist. Then, due to a division by zero, a breakdown will occur in the Lanczos type algorithms described in Section 4. It is possible to avoid such a breakdown by jumping over the nonexisting polynomials and considering only the existing ones which are usually called regular. We shall now change a little bit our notations and call $P_{k}$ and $P_{k+1}$ two successive regular orthogonal polynomials with respect to $c$, of respective degrees $n_{k}$ and $n_{k+1}=n_{k}+m_{k}$ at most. Similarly, $P_{k}^{(1)}$ and $P_{k+1}^{(1)}$ will denote two successive regular monic orthogonal polynomials with respect to $c^{(1)}$, of respective degrees $n_{k}$ and $n_{k+1}$.

It was proved by Draux [10] that $P_{k}^{(1)}$ satisfies the conditions

$$
\begin{aligned}
c^{(1)}\left(\xi^{i} P_{k}^{(1)}\right) & =c\left(\xi^{i+1} P_{k}^{(1)}\right)=0 \quad \text { for } i=0, \ldots, n_{k}+m_{k}-2, \\
c^{(1)}\left(\xi^{n_{k}+m_{k}-1} P_{k}^{(1)}\right) & =c\left(\xi^{n_{k}+m_{k}} P_{k}^{(1)}\right) \neq 0 .
\end{aligned}
$$


These conditions determine the length $m_{k}$ of the jump. Moreover, the polynomials $P_{k}^{(1)}$ satisfy the recurrence relationship

$$
P_{k+1}^{(1)}(\xi)=q_{k}(\xi) P_{k}^{(1)}(\xi)-C_{k+1} P_{k-1}^{(1)}(\xi), \quad k=0,1, \ldots
$$

with $P_{-1}^{(1)}(\xi)=0, P_{0}^{(1)}(\xi)=1$ and $q_{k}$ a monic polynomial of degree $m_{k}$.

It can also be proved that, in such a case,

$$
P_{k+1}(\xi)=P_{k}(\xi)-\xi w_{k}(\xi) P_{k}^{(1)}(\xi)
$$

with $P_{0}(\xi)=1$ and $w_{k}$ a polynomial of degree $m_{k}-1$ at most.

The coefficients of the polynomials $q_{k}$ and $w_{k}$ and the constants $C_{k+1}$ are obtained by imposing the orthogonality conditions on the respective polynomials.

Gathering all these formulae together we have the following algorithm called the MRZ (Method of Recursive Zoom) [6]:

- Choose $x_{0}$ and $y$. Set

$$
r_{0}=z_{0}=b-A x_{0}, \quad z_{-1}=0, \quad n_{0}=0 .
$$

- For $k=0,1, \ldots$ compute $m_{k}$ and then if $n_{k}+m_{k} \leq n$ set

$$
x_{k+1}=x_{k}+w_{k}(A) z_{k}, \quad r_{k+1}=r_{k}-A w_{k}(A) z_{k} .
$$

- If $r_{k+1} \neq 0$, then compute

$$
n_{k+1}=n_{k}+m_{k}, \quad z_{k+1}=q_{k}(A) z_{k}-C_{k+1} z_{k-1} .
$$

Clearly this algorithm is a generalization of the Lanczos/Orthodir and BIODIR algorithms. It cannot suffer from breakdown except the incurable hard one which occurs if $c\left(\xi^{n} P_{k}^{(1)}\right)=0$. The corresponding subroutine is given in [7].

If we make the supplementary assumption that $c\left(\xi^{n_{k}} P_{k}\right) \neq 0$ then it is possible to generalize some of the other algorithms given in Section 4.

As explained above, breakdown is due to the non-existence of some polynomials $P_{k}$ and the remedy is to jump over these non-existing polynomials. In the methods Lanczos/Orthores and Lanczos/Orthomin, we made the supplementary assumption that $P_{k}$ has exact degree $k$. In fact this assumption is totally unnecessary in the theory of the Lanczos method but it was required by the form of the recurrence relation used, thus being a supplementary (and unnecessary) cause for breakdown.

Now if $\left|c^{(1)}\left(\xi^{n_{k}+m_{k}-1} P_{k}^{(1)}\right)\right|$ is different from zero but small (and possibly badly computed) the coefficients of the recurrence relations given in Section 4 could be large and badly computed and rounding errors could affect the algorithm. The same is true if the quantities $\left|c^{(1)}\left(\xi^{i} P_{k}^{(1)}\right)\right|$ are not zero for $i=n_{k}, \ldots, n_{k}+$ $m_{k}-2$ but small; in that case no breakdown occurs in the method but numerical instability will be present, a situation called near-breakdown.

It is possible to avoid such a near-breakdown by jumping over these polynomials which could be badly computed and to compute directly the first regular polynomial following them. 
Thus, let $\varepsilon \geq 0$ be given. We define $m_{k} \geq 1$ such that

$$
\begin{array}{ll}
\left|c^{(1)}\left(\xi^{i} P_{k}^{(1)}\right)\right| \leq \varepsilon & \text { for } i=n_{k}, \ldots, n_{k}+m_{k}-2, \\
\left|c^{(1)}\left(\xi^{i} P_{k}^{(1)}\right)\right|>\varepsilon & \text { for } i=n_{k}+m_{k}-1 .
\end{array}
$$

Let $n_{k+1}=n_{k}+m_{k}$. We shall denote by $P_{k+1}^{(1)}$ the regular orthogonal polynomial of degree $n_{k+1}$ with respect to $c^{(1)}$. As explained in the sequel if such a polynomial does not exist (and we are able to detect such a case) the value of $m_{k}$ has to be increased until a regular polynomial has been obtained. We shall denote by $P_{k+1}$ the corresponding orthogonal polynomial of degree $n_{k+1}$ at most with respect to $c$ normalized by the condition $P_{k+1}(0)=1$.

Let us first compute $P_{k+1}$. We shall write it in the form

$$
P_{k+1}(\xi)=P_{k}(\xi)-\xi w_{k}(\xi) P_{k}^{(1)}(\xi)-\xi v_{k}(\xi) P_{k}(\xi)
$$

where $w_{k}$ is a polynomial of degree at most $m_{k}-1$ and $v_{k}$ a polynomial of degree at most $m_{k}-2$ if $n_{k}-m_{k}+1 \geq 0$ and of degree at most $n_{k}-1$ if $n_{k}-m_{k}+1<0$.

Let us recall that, in the case of a breakdown, $v_{k}$ is identically zero. form

Let us now try to compute recursively $P_{k+1}^{(1)}$. We shall look for $P_{k+1}^{(1)}$ in the

$$
P_{k+1}^{(1)}(\xi)=q_{k}(\xi) P_{k}^{(1)}(\xi)+t_{k}(\xi) P_{k}(\xi)
$$

where $q_{k}$ is a monic polynomial of degree $m_{k}$ and $t_{k}$ a polynomial of degree at most $m_{k}-1$ if $n_{k}-m_{k} \geq 0$ and of degree at most $n_{k}-1$ otherwise.

If $c\left(\xi^{n_{k}} P_{k}\right)=0$ this system is singular. However, this case will seldom occur and most of the time $\left|c\left(\xi^{n_{k}} P_{k}\right)\right|$ will not be equal to zero but small. Such a case can also be avoided.

If the systems giving the coefficients of the above polynomials are singular, it means that $P_{k+1}^{(1)}$ does not exist and the value of $m_{k}$ has to be increased until a regular polynomial $P_{k+1}^{(1)}$ has been obtained.

Setting $r_{k}=P_{k}(A) r_{0}$ and $z_{k}=P_{k}^{(1)}(A) r_{0}$, we obtain an algorithm for avoiding near-breakdown in the Lanczos method; it was called the BSMRZ, [7].

6. The CGS. A variant of the Lanczos method was proposed by Sonneveld [15]. It is the so-called conjugate gradient squared method (CGS for short) which consists in taking

$$
r_{k}=P_{k}^{2}(A) r_{0}
$$

This method obviously has the same finite convergence property as the Lanczos method. From the above formulae it is obviously possible to avoid also breakdown and near-breakdown in the CGS algorithm by squaring the above formulae. This is done in [9] for the breakdown and in [5] for the near-breakdown. As an example let us show how to treat the case of near-breakdown. We first take the relation 
(8) of the BSMRZ and square it. We obtain

$$
P_{k+1}^{2}=\left(1-\xi v_{k}\right)^{2} P_{k}^{2}-2\left(1-\xi v_{k}\right) \xi w_{k} P_{k} P_{k}^{(1)}+\xi^{2} w_{k}^{2} P_{k}^{(1)^{2}} .
$$

For using this relation, it is necessary to compute recursively the polynomials $P_{k+1}^{(1)^{2}}$ and $P_{k+1} P_{k+1}^{(1)^{2}}$. Thus, let us square the relation (9) of the BSMRZ. We obtain

$$
P_{k+1}^{(1)^{2}}=q_{k}^{2} P_{k}^{(1)^{2}}+2 q_{k} t_{k} P_{k} P_{k}^{(1)}+t_{k}^{2} P_{k}^{2}
$$

Finally, multiplying (8) by (9) leads to

$$
P_{k+1} P_{k+1}^{(1)}=\left(q_{k}-\xi q_{k} v_{k}-\xi t_{k} w_{k}\right) P_{k} P_{k}^{(1)}-\xi q_{k} w_{k} P_{k}^{(1)^{2}}+t_{k}\left(1-\xi v_{k}\right) P_{k}^{2}
$$

Thus, if we set

$$
r_{k}=P_{k}^{2}(A) r_{0}, \quad z_{k}=P_{k}^{(1)^{2}}(A) r_{0}, \quad s_{k}=P_{k}(A) P_{k}^{(1)}(A) r_{0},
$$

then we obtain the following algorithm called, for obvious reasons, the BSMRZS:

$$
\begin{aligned}
r_{k+1}= & \left(I-A v_{k}(A)\right)^{2} r_{k}-2\left(I-A v_{k}(A)\right) A w_{k}(A) s_{k}+A^{2} w_{k}^{2}(A) z_{k}, \\
x_{k+1}= & x_{k}-\left(A v_{k}(A)-2 I\right) v_{k}(A) r_{k}+2\left(I-A v_{k}(A)\right) w_{k}(A) s_{k}-A w_{k}^{2}(A) z_{k}, \\
z_{k+1}= & q_{k}^{2}(A) z_{k}+2 q_{k}(A) t_{k}(A) s_{k}+t_{k}^{2}(A) r_{k}, \\
s_{k+1}= & \left(q_{k}(A)-A q_{k}(A) v_{k}(A)-A t_{k}(A) w_{k}(A)\right) s_{k}-A q_{k}(A) w_{k}(A) z_{k} \\
& +t_{k}(A)\left(I-A v_{k}(A)\right) r_{k} .
\end{aligned}
$$

\section{References}

[1] C. Brezinski, Padé-type Approximation and General Orthogonal Polynomials, Internat. Ser. Number. Math. 50, Birkhäuser, Basel 1980.

[2] - - The methods of Vorobyev and Lanczos, to appear.

[3] —, Biorthogonality and its Applications to Numerical Analysis, Marcel Dekker, New York 1992 .

[4] C. Brezinski and M. Redivo Zaglia, A new presentation of orthogonal polynomials with application to their computation, Numer. Algorithms 1 (1991), 207-221.

[5] —, - - Treatment of near-breakdown in the CGS algorithm, to appear.

[6] C. Brezinski, M. Redivo Zaglia and H. Sadok, A breakdown-free Lanczos type algorithm for solving linear systems, Numer. Math. 63 (1992), 29-38.

[7] —, - - - Avoiding breakdown and near-breakdown in Lanczos type algorithms, Numer. Algorithms 1 (1991), 261-284

[8] C. Brezinski and H. Sadok, Lanczos type methods for solving systems of linear equations, Appl. Numer. Math., to appear.

[9] _- - Avoiding breakdown in the CGS algorithm, Numer. Algorithms 1 (1991), 199-206.

[10] A. Draux, Polynômes Orthogonaux Formels - Applications, Lecture Notes in Math. 974, Springer, Berlin 1983.

[11] R. Fletcher, Conjugate gradient methods for indefinite systems, in: Numerical Analysis, G. A. Watson (ed.), Lecture Notes in Math. 506, Springer, Berlin 1976, 73-89.

[12] M. H. Gutknecht, A completed theory of the unsymmetric Lanczos process and related algorithms. Part I, SIAM J. Matrix Anal. Appl. 13 (1992), 594-639. 
[13] M. R. Hestenes and E. Stiefel, Methods of conjugate gradients for solving linear systems, J. Res. Nat. Bur. Standards 49 (1952), 409-439.

[14] C. Lanczos, Solution of systems of linear equations by minimized iterations, ibid., 33-53.

[15] P. Sonneveld, CGS, a fast Lanczos-type solver for nonsymmetric linear systems, SIAM J. Sci. Statist. Comput. 10 (1989), 36-52.

[16] Yu. V. Vorobyev, Method of Moments in Applied Mathematics, Gordon and Breach, New York 1965.

[17] D. M. Young and K. C. Jea, Generalized conjugate-gradient acceleration of nonsymmetrizable iterative methods, Linear Algebra Appl. 34 (1980), 159-194. 